Bangladesh J. Plant Taxon. 18(2): 177-197, 2011 (December)

(C) 2011 Bangladesh Association of Plant Taxonomists

\title{
UPDATED NOMENCLATURE AND TAXONOMIC STATUS OF THE PLANTS OF BANGLADESH INCLUDED IN HOOK. F., THE FLORA OF BRITISH INDIA: VOLUME-I
}

\author{
M. EnAmur RASHid AND M. Atiqur RAHMAN ${ }^{*}$ \\ Department of Botany, University of Chittagong, Chittagong 4331, Bangladesh
}

Keywords: J.D. Hooker; Flora of British India; Bangladesh; Nomenclature; Taxonomic status.

\begin{abstract}
Sir Joseph Dalton Hooker in his first volume of the Flora of British India includeed a total of 2460 species in 452 genera under 44 natural orders (= families) of which a total of 226 species in 114 genera under 33 natural orders were from the area now in Bangladesh. These taxa are listed with their updated nomenclature and taxonomic status as per ICBN following Cronquist's system of plant classification. The current number recognized, so far, are 220 species in 131 genera under 44 families. The recorded area in Bangladesh and the name of specimen's collector, as in Hook.f., are also provided.
\end{abstract}

\section{Introduction}

J.D. Hooker compiled his first volume of the "Flora of British India" with three parts published in 3 different dates. Each part includes a number of natural orders. Part I includes the natural order Ranunculaceae to Polygaleae while Part II includes Frankeniaceae to Geraniaceae and Part III includes Rutaceae to Sapindaceae. Hooker was assisted by various botanists in describing the taxa of 44 natural orders of this volume. Altogether 10 contributors including J.D. Hooker were involved in this volume. Publication details along with number of cotributors and distribution of taxa of 3 parts of this volume are mentioned in Table 1.

This volume includes a total of 44 natural orders (now treated as families), 98 tribes, 452 genera and 2460 species. Hooker described 5 natural orders by himself, 14 natural orders jointly with T. Thomson, 3 natural orders with M.P. Edgeworth, 1 natural order with T. Anderson while Maxwell T. Masters, Alfred W. Bennett, W.T. Thiselton Dyer, W.P. Hiern, T. Anderson and M.A. Lawson individually described remaining 21 natural orders. Out of these 44 natural orders, 33 were recorded with 226 species from the area now in Bangladesh. These natural orders of the volume-I and their contributors are listed below in Table 2 with distribution of taxa.

\section{Materials and Methods}

The first volume of the Flora of British India (Hooker, 1872) was considered as an initial step to prepare a list of species which have been recorded in it from the area now in Bangladesh.

The recorded area (collection locality), collector's name, etc. were determined by checking the protologue of each species carefully. The recorded localities, as mentioned in the protologues, were confirmed by consulting Roxburgh $(1814,1832)$ and Wallich (1828-49) which appeared before the Flora of British India. Moreover, literature including Kurz (1877), Prain (1903), Heinig

*Corresponding author. E-mail: atiquerahman125@hotmail.com 
(1925), Cowan (1926) and Sinclair (1956) appeared after the Flora of British India, were also consulted to ascertain the corresponding places of occurrence in the area of Bangladesh.

Table 1. Publication details of Flora of British India, Volume 1.

\begin{tabular}{lccccc}
\hline Published parts & $\begin{array}{c}\text { Year of } \\
\text { publication }\end{array}$ & $\begin{array}{c}\text { No. of } \\
\text { contributors }\end{array}$ & $\begin{array}{c}\text { No. of natural } \\
\text { orders }\end{array}$ & $\begin{array}{c}\text { No. of } \\
\text { genera }\end{array}$ & No. of species \\
\hline Part-I & 1872 & 4 & 16 & 171 & 777 \\
Part-II & 1874 & 5 & 16 & 139 & 929 \\
Part-III & 1875 & 5 & 12 & 142 & 754 \\
\hline
\end{tabular}

The current nomenclature of each species was determined as per ICBN by consulting Voss (1983) and by searching Internet sources (I-VI), and taxonomic status were determined following Cronquist's (1981) system of plant classification. For currect author citation Brummitt and Powell (1992) was consulted. Synonyms were checked by consulting recently published relevant literature, viz., Khan (1972-1987), Hara and Williamms (1979), Grierson and Long (1984, 1991), Brummitt (1992), Sharma et al. (1993), Sharma and Balakrishnan (1993), Sharma and Sanjapa (1993), Mabberley (1997), Hajra et al. (1997), Hajra (1997), Press et al. (2000), Khan and Rahman (1989-2002), Rahman et al. (2003), Khanam and Ara (2007), and Ahmed et al. (2008a,b;2009a,b,c).

Table 2. Natural Orders with contributors and distribution of taxa in the Volume-I

\begin{tabular}{llccc}
\hline $\begin{array}{l}\text { Natural order } \\
\text { as in Volume-I }\end{array}$ & Name of Contributors & $\begin{array}{c}\text { No. of } \\
\text { genera }\end{array}$ & $\begin{array}{c}\text { No. of } \\
\text { species }\end{array}$ & $\begin{array}{c}\text { No. of species from the } \\
\text { area of Bangladesh }\end{array}$ \\
\hline 1. Ranunculaceae & J.D. Hooker \& T. Thomson & 19 & 121 & 2 \\
2. Dilleniaceae & J.D. Hooker \& T. Thomson & 6 & 34 & 3 \\
3. Magnoliaceae & J.D. Hooker \& T. Thomson & 8 & 27 & 2 \\
4. Anonaceae & J.D. Hooker \& T. Thomson & 26 & 194 & 22 \\
5. Menispermaceae & J.D. Hooker \& T. Thomson & 19 & 34 & 10 \\
6. Berberideae & J.D. Hooker \& T. Thomson & 6 & 17 & 0 \\
7. Nymphaeaceae & J.D. Hooker \& T. Thomson & 5 & 8 & 1 \\
8. Papaveraceae & J.D. Hooker \& T. Thomson & 5 & 13 & 0 \\
9. Fumariaceae & J.D. Hooker \& T. Thomson & 4 & 31 & 0 \\
10. Cruciferae & J.D. Hooker \& T. Anderson & 43 & 138 & 1 \\
11. Capparideae & J.D. Hooker \& T. Thomson & 8 & 56 & 1 \\
12. Resedaceae & J.D. Hooker \& T. Thomson & 3 & 4 & 0 \\
13.Violaceae & J.D. Hooker \& T. Thomson & 3 & 26 & 3 \\
14. Bixineae & J.D. Hooker \& T. Thomson & 10 & 26 & 4 \\
15. Pittosporeae & J.D. Hooker \& T. Thomson & 1 & 9 & 0 \\
16. Polygaleae & Alfred W. Bennett & 5 & 39 & 3 \\
17. Frankeniaceae & M.P. Edgeworth \& J.D. Hooker & 1 & 1 & 0 \\
18. Caryophylleae & W.T. Thiselton Dyer & 19 & 108 & 0 \\
\hline & & & & 1 \\
& & & \\
\end{tabular}


Table 2 contd.

\begin{tabular}{|c|c|c|c|c|}
\hline $\begin{array}{l}\text { Natural order } \\
\text { as in Volume-I }\end{array}$ & Name of Contributors & $\begin{array}{l}\text { No. of } \\
\text { genera }\end{array}$ & $\begin{array}{l}\text { No. of } \\
\text { species }\end{array}$ & $\begin{array}{l}\text { No. of species from the } \\
\text { area of Bangladesh }\end{array}$ \\
\hline 19. Portulaceae & W.T. Thiselton Dyer & 2 & 6 & 0 \\
\hline 20. Tamariscineae & W.T. Thiselton Dyer & 2 & 8 & 0 \\
\hline 21. Elatineae & W.T. Thiselton Dyer & 2 & 6 & 0 \\
\hline 22. Hypericineae & W.T. Thiselton Dyer & 3 & 26 & 3 \\
\hline 23. Guttiferae & T. Anderson & 6 & 65 & 10 \\
\hline 24.Ternstrcemiaceae & W.T. Thiselton Dyer & 14 & 56 & 7 \\
\hline 25. Dipterocarpeae & W.T. Thiselton Dyer & 9 & 95 & 11 \\
\hline 26. Malvaceae & Maxwell T. Masters & 27 & 127 & 9 \\
\hline 27.Sterculiaceae & Maxwell T. Masters & 17 & 90 & 12 \\
\hline 28.Tiliaceae & Maxwell T. Masters & 13 & 115 & 22 \\
\hline 29. Linea & J.D. Hooker & 7 & 23 & 2 \\
\hline 30. Malpighiaceae & J.D. Hooker & 3 & 14 & 2 \\
\hline 31. Zygophylleae & M.P. Edgeworth \& J.D. Hooker & 4 & 9 & 0 \\
\hline 32. Geraniaceae & M.P. Edgeworth \& J.D. Hooker & 10 & 180 & 3 \\
\hline 33. Rutaceae & J.D. Hooker & 23 & 95 & 14 \\
\hline 34. Simarubeae & Alfred W. Bennett & 9 & 17 & 1 \\
\hline 35. Ochnaceae & Alfred W. Bennett & 4 & 13 & 1 \\
\hline 36. Burseraceae & Alfred W. Bennett & 10 & 39 & 3 \\
\hline 37. Meliaceae & W.P. Hiern & 19 & 85 & 9 \\
\hline 38. Chailletiaceae & J.D. Hooker & 1 & 6 & 1 \\
\hline 39. Olacineae & Maxwell T. Masters & 23 & 69 & 10 \\
\hline 40. Ilicineae & J.D. Hooker & 1 & 26 & 2 \\
\hline 41.Celastrineae & M.A. Lawson & 13 & 114 & 13 \\
\hline 42. Rhamneae & M.A. Lawson & 12 & 57 & 5 \\
\hline 43. Ampelideae & M.A. Lawson & 3 & 101 & 23 \\
\hline 44. Sapindaceae & W.P. Hiern & 24 & 132 & 11 \\
\hline Total: 44 & 10 & 452 & 2460 & 226 \\
\hline
\end{tabular}

\section{Results and Discusion}

The search of the first volume of the Flora of British India revealed a total of 226 species under 114 genera and 33 natural orders from the area now in Bangladesh. After current nomenclatural treatment, the number of species reduced to 220 . While the genera and families splited to 131 and 44, respectively. It is determined, so far, that 35 generic names are changed and 79 remain unchanged. On the other hand, 106 species names are changed and 120 remain unchanged. Hence, 220 species and 131 genera under 44 families. are recognized, so far, from the area of Bangladesh and presented in Table 3. 
Table 3. List of Taxa as in Hook.f., the Flora of British India (FBI), Volume-I from the area of Bangladesh with their current nomenclature and taxonomic status.

Name of species, Natural Order as in Hook.f. with recorded area and collector's name

1. Clematis cadmia Ham. ex Wall. Natural Order: Ranunculaceae Eastern Bengal - Griffith

2. Naravelia zylanica DC. Natural Order: Ranunculaceae Bengal - Wall. Cat. 4687

3. Tetracera assa DC. Natural Order: Dilleniaceae Chittagong - Wall. Cat. 6629

4. Dillenia indica Linn. Natural Order: Dilleniaceae Silhet - Wall. Cat. 943

5. Dillenia scabrella Roxb. Natural Order: Dilleniaceae Silhet - Wall. Cat. 944

6. Magnolia sphenocarpa Roxb. Natural Order: Magnoliaceae Chittagong - Wall. Cat.975

7. Kadsura Roxburghiana Arn. Natural Order: Magnoliaceae Silhet - Wall. Cat. 4987

8. Uvaria bracteata Roxb. Natural Order: Anonaceae Silhet - Roxburgh

9. Uvaria macrophylla Roxb. Natural Order: Anonaceae Silhet - Wall. Cat. 6487

10. Artabotrys suaveolens Blume Natural Order: Anonaceae Silhet - Wall. Cat. 6416

11. Unona dunalii Wall. Natural Order: Anonaceae Chittagong, Sitakund - H. f. \& T.

12. Unona dumosa Roxb. Natural Order: Anonaceae Silhet - Roxburgh

13. Unona discolor Vahl Natural Order: Anonaceae Silhet - Wall. Cat. 6420 (partly)

14. Unona longiflora Roxb. Natural Order: Anonaceae Chittagong - Wall. Cat. 6419
Current nomenclature with loc. cite., and

family as Cronquist (1981)

1. Clematis cadmia Buch.-Ham. ex Hook.f. \& Thom., Fl. Ind.: 5 (1855).

Family: Ranunculaceae

2. Naravelia zylanica (L.) DC., Syst. Nat. 1: 167 (1817). Family: Ranunculaceae

3. Tetracera indica (Houtt. ex Christm. \& Panz.) Merr., Interpr. Rump. Herb. Amboin.: 367 (1917). Family: Dilleniaceae

4. Dillenia indica L., Sp. Pl.: 535 (1753).

Family: Dilleniaceae

5. Dillenia scabrella Roxb. ex Wall. in Pl. As. Rar. 1: 20, t. 22 (1830). Family: Dilleniaceae

6. Magnolia pterocarpa Roxb., Pl. Corom. 3: 62, t. 266 (1820). Family: Magnoliaceae

7. Kadsura heteroclita (Roxb.) Craib., Fl. Siam. Enum.1: 28 (1925). Family: Schisandraceae

8. Cyathostemma argenteum (Bl.) J. Sinclair in Sarawak Mus. Journ. 5: 3: 599 (1951).

Family: Annonaceae

9. Uvaria cordata (Dunal) Alston in Handb. Fl. Ceyl. Suppl. 6: 4 (1931). Family: Annonaceae

10. Artabotrys suaveolens Blume in Fl. Jav. Anon. p. 62. t. 30 \& 31D (1830). Family: Annonaceae

11. Desmos dunalii (Hook.f. \& Thom.) Saff. in Bull. Torr. Bot. Club 39: 506 (1912).

Family: Annonaceae

12. Desmos dumosus (Roxb.) Saff. in Bull. Torr.

Bot. Club 39:506 (1912). Family: Annonaceae

13. Desmos chinensis Lour., Fl. Cochinch. 1: 352 (1790). Family: Annonaceae

14. Desmos longiflorus (Roxb.) Saff. in Bull. Torr. Bot. Club 39: 507 (1912). Family: Annonaceae 
Table 3 contd.

\begin{tabular}{|c|c|}
\hline $\begin{array}{l}\text { Name of species, Natural Order as in Hook.f. } \\
\text { with recorded area and collector's name }\end{array}$ & $\begin{array}{l}\text { Current nomenclature with loc. cite., and } \\
\text { family as Cronquist (1981) }\end{array}$ \\
\hline $\begin{array}{l}\text { 15. Polyalthia cerasoides Benth. } \\
\text { Natural Order: Anonaceae } \\
\text { Eastern exposer - Hamilton }\end{array}$ & $\begin{array}{l}\text { 15. Polyalthia cerasoides (Roxb.) Bedd.in Ic. Pl. Ind. } \\
\text { Orien.: } 17 \text { (1869). Family: Annonaceae }\end{array}$ \\
\hline $\begin{array}{l}\text { 16. Polyalthia simiarum Benth. \& H. f. } \\
\text { Natural Order: Anonaceae } \\
\text { Silhet - Hamilton and Wallich }\end{array}$ & $\begin{array}{l}\text { 16. Polyalthia simiarum (Buch-Ham. ex Hook. f.) } \\
\text { Hook.f. \& Thom. in Fl. Brit. Ind. 1: } 63 \text { (1872). } \\
\text { Family: Annonaceae }\end{array}$ \\
\hline $\begin{array}{l}\text { 17. Polyalthia jenkinsii Benth. \& H. f. } \\
\text { Natural Order: Anonaceae } \\
\text { Silhet - Kurz }\end{array}$ & $\begin{array}{l}\text { 17. Polyalthia jenkinsii Hook.f. \& Thom. in Fl. Brit. } \\
\text { Ind. 1: } 64 \text { (1872). Family: Annonaceae }\end{array}$ \\
\hline $\begin{array}{l}\text { 18. Polyalthia suberosa Benth. \& H. f. } \\
\text { Natural Order: Anonaceae } \\
\text { Bengal - not mentioned }\end{array}$ & $\begin{array}{l}\text { 18. Polyalthia suberosa (Roxb.) Thw. in Enum. Pl. } \\
\text { Zeyl.: } 398 \text { (1864). Family: Annonaceae }\end{array}$ \\
\hline $\begin{array}{l}\text { 19. Polyalthia argentea H. f. \& T. } \\
\text { Natural Order: Anonaceae } \\
\text { Silhet - H. f. \& T. }\end{array}$ & $\begin{array}{l}\text { 19. Trivalvaria argentea (Hook.f. \& Thom.) J. } \\
\text { Sinclair in Sarawak Mus. Journ. 5: (1951). } \\
\text { Family: Annonaceae }\end{array}$ \\
\hline $\begin{array}{l}\text { 20. Oxymitra fornicata } \text { H. f. \& T. } \\
\text { Natural Order: Anonaceae } \\
\text { Silhet - Wall. Cat. 6423A }\end{array}$ & $\begin{array}{l}\text { 20. Friesodielsia fornicata (Roxb.) Das in Bull. Bot. } \\
\text { Sunv. Ind. 5: } 43 \text { (1963). } \\
\text { Family: Annonaceae }\end{array}$ \\
\hline $\begin{array}{l}\text { 21. Mitrephora tomentosa H. f. \& T. } \\
\text { Natural Order: Anonaceae } \\
\text { Chittagong - H. f. \& T. }\end{array}$ & $\begin{array}{l}\text { 21. Mitrephora tomentosa Hook.f. \& Thom., Fl. Ind. } \\
\text { p. } 113 \text { (1855). Family: Annonaceae }\end{array}$ \\
\hline $\begin{array}{l}\text { 22. Anona reticulata Linn. } \\
\text { Natural Order: Anonaceae } \\
\text { Naturalized in Bengal }\end{array}$ & $\begin{array}{l}\text { 22. Anona reticulata L., Sp. Pl.: } 537 \text { (1753). } \\
\text { Family: Annonaceae }\end{array}$ \\
\hline $\begin{array}{l}\text { 23. Melodorum rubiginosum H. f. \& T. } \\
\text { Natural Order: Anonaceae } \\
\text { Chittagong and Silhet - H. f. \& T. }\end{array}$ & $\begin{array}{l}\text { 23. Fissistigma rubiginosum (A. DC.) Merr. in } \\
\text { Philipp. Journ. Sc. Bot. 15: } 135 \text { (1919). } \\
\text { Family: Annonaceae }\end{array}$ \\
\hline $\begin{array}{l}\text { 24.Melodorum polyanthum H. f. \& T. } \\
\text { Natural Order: Anonaceae } \\
\text { Silhet - Wall. Cat. } 6467\end{array}$ & $\begin{array}{l}\text { 24. Fissistigma polyanthum (Hook.f. \& Thom.) Merr. } \\
\text { in Philipp. Journ. Sc. Bot. 15:135 (1919). } \\
\text { Family: Annonaceae }\end{array}$ \\
\hline $\begin{array}{l}\text { 25. Melodorum rufinerve H. f. \& T. } \\
\text { Natural Order: Anonaceae } \\
\text { Silhet - H. f. \& T. }\end{array}$ & $\begin{array}{l}\text { 25. Fissistigma rufinerve (Hook.f. \& Thom.) Merr. in } \\
\text { Philipp. Journ. Sc. Bot. 15: } 136 \text { (1919). } \\
\text { Family: Annonaceae }\end{array}$ \\
\hline $\begin{array}{l}\text { 26. Melodorum Wallichii H. f. \& T. } \\
\text { Natural Order: Anonaceae } \\
\text { Silhet - Wallich }\end{array}$ & $\begin{array}{l}\text { 26. Fissistigma wallichii (Hook. f. \& Thom.) Merr. } \\
\text { in Philipp. Journ. Sc. Bot. 15: } 137 \text { (1919). } \\
\text { Family: Annonaceae }\end{array}$ \\
\hline $\begin{array}{l}\text { 27. Saccopetalum longiflorum H. f. \& T. } \\
\text { Natural Order: Anonaceae } \\
\text { Eastern Bengal - Hamilton }\end{array}$ & $\begin{array}{l}\text { 27. Miliusa longiflora (Hook. f. \& Thom.) Finet \& } \\
\text { Gagnep. in Bull. Soc. Bot. Fr. 53(4): } 153 \text { (1906). } \\
\text { Family: Annonaceae }\end{array}$ \\
\hline $\begin{array}{l}\text { 28. Alphonsia lutea H. f. \& T. } \\
\text { Natural Order: Anonaceae } \\
\text { Silhet - Beddome }\end{array}$ & $\begin{array}{l}\text { 28. Alphonsia lutea (Roxb.) Hook.f. \& Thom., Fl. Ind. } \\
\text { p.153 (1855). Family: Annonaceae }\end{array}$ \\
\hline
\end{tabular}


Table 3 contd.

Name of species, Natural Order as in Hook.f. with recorded area and collector's name

29. Alphonsia ventricosa H. f. \& T. Natural Order: Anonaceae Chittagong - Wall. Cat. 6453

30. Parabaena sagittata Miers Natural Order: Menispermaceae Chittagong - Wall. Cat. 4984

31. Tinospora malabarica Miers Natural Order: Menispermaceae Chittagong - Wall. Cat. 4969

32. Tinospora crispa Miers Natural Order: Menispermaceae Silhet - Wall. Cat. 49966A, B.

33. Tinospora tomentosa Miers Natural Order: Menispermaceae Bengal - Roxburgh

34. Anamirta cocculus W. \& A. Natural Order: Menispermaceae Eastern Bengal - Wall. Cat. 4954

35. Tiliacora racemosa Coleb. Natural Order: Menispermaceae Bengal - not mentioned

36. Limacia cuspidata H. f. \& T. Natural Order: Menispermaceae Eastern Bengal - Wall. Cat. 4960

37. Pericampylus incanus Miers Natural Order: Menispermaceae Chittagong - Wall. Cat. 4980

38. Stephania hernandifolia Walp. Natural Order: Menispermaceae Chittagong - Wall. Cat. 4977 D-H,K

39. Pycnarrhena pleniflora Miers. Natural Order: Menispermaceae Silhet - Wallich

40. Euryale ferox Salisb. Natural Order: Nymphaeaceae Jheels of Eastern Bengal

41. Nasturtium palustre DC. Natural Order: Cruciferae Bengal - not mentioned

42. Capparis horrida Linn. $\mathrm{f}$. Natural Order: Capparideae Chittagong - Wall. Cat. 6981
Current nomenclature with loc. cite., and

family as Cronquist (1981)

29. Alphonsia ventricosa (Roxb) Hook.f. \& Thom., Fl. Ind. p. 152 (1855).

Family: Annonaceae

30. Parabaena sagittata Miers ex Hook.f. \& Thom., Fl. Ind. p. 181 (1855).

Family: Menispermaceae

31. Tinospora sinensis (Lour.) Merr., Sunyasenia 1: 193 (1934). Family: Menispermaceae

32. Tinospora crispa (L.) Hook. f. \& Thom., Fl. Ind. p. 183 (1855). Family: Menispermaceae

31. Tinospora sinensis (Lour.) Merr., Sunyasenia 1: 193 (1934). Family: Menispermaceae

33. Anamirta cocculus (L.) Wight \& Arn., Prod. 1:446 (1834). Family: Menispermaceae

34. Tiliacora acuminata (Lam.) Hook.f. \& Thom., Fl. Ind. p.187 (1855). Family: Minispermaceae

35. Hypserpa nitida Miers. in Hook.f., Kew Journ. Bot. 3: 258 (1851). Family: Minispermaceae

36. Pericampylus glaucus (Lam.) Merr., Interp. Rump.. Herb. Amb.: 219 (1917).

Family: Menispermaceae

37. Stephania japonica (Thunb.) Miers in Ann. Mag. Nat. Hist. Ser. 3. 18: 14 (1866). var. timoriensis (DC.) Forman, Kew Bull. 11: 55 (1956). Family: Menispermaceae

38. Pycnarrhena pleniflora (planiflora) Hook.f. \& Thom., Fl. Ind. p. 206 (1855).

Family: Menispermaceae

39. Euryale ferox Salisb. in Kon. \& Sims. in Ann.Bot. 2: 74 (1806). Family: Nymphaeaceae

40. Rorippa palustris (L.) Bess. in Enum. Pl. Volhyniae: 27 (1822). Family: Brassicaceae

41. Capparis zeylanica L., Sp. Pl. ed. 2: 720 (1762). Family: Capparaceae 
Table 3 contd.

Name of species, Natural Order as in Hook.f. with recorded area and collector's name

43. Ionidium suffruticosum Ging. Natural Order: Violaceae Bengal - Wall. Cat. 1439

44. Alsodeia bengalensis Wall. Natural Order: Violaceae Silhet - Wallich and Griffith

45. Alsodeia roxburghii Wall. Natural Order: Violaceae Silhet - Wallich

46. Flacourtia inermis Roxb. Natural Order: Bixineae Silhet - Jack \& c.

47. Flacourtia Cataphracta Roxb. Natural Order: Bixineae Chittagong - Wall. Cat. 6674

48. Flacourtia sepiaria Roxb. Natural Order: Bixineae Throughout Bengal

49. Gynocardia odorata R. Br. Natural Order: Bixineae Chittagong - not mentioned

50. Salomonia cantoniensis Lour. Natural Order: Polygaleae Eastern Bengal - Wall. Cat. 4192

51. Securidaca Tavoyana Wall. Natural Order: Polygaleae Chittagong, Silhet -Wall. Cat. 4196

52. Xanthophyllum flavescens Roxb. Natural Order: Polygaleae Chittagong, Silhet - not mentioned

53. Hypericum japonicum Thunb. Natural Order: Hypericineae Silhet - Wall. Cat. 4811

54. Hypericum breviflorum Wall. Natural Order: Hypericineae Silhet - Wallich

55. Cratoxylon neriifolium Kurz Natural Order: Hypericineae Chittagong - H. f. \& T.

56. Garcinia cornea Linn. Natural Order: Guttiferae Silhet - Wall. Cat. 4852
Current nomenclature with loc. cite., and family as Cronquist (1981)

42. Hybanthus enneaspermus (L.) F. Muell., Fragm. Phyt. Austr. 10: 81 (1876). Family: Violaceae

43. Rinorea bengalensis (Wall.) O. Kutze., Rev. Gen. Pl. 1: 42 (1891). Family: Violaceae

44. Rinorea heteroclita (Roxb.) Craib. in Fl. Siam. Enum. 1:89 (1925). Family: Violaceae

45. Flacourtia inermis Roxb., Pl. Corom. 3: 16, t. 222 (1811). Family: Flacourtiaceae

46. Flacourtia jangomas (Lour.) Raeusch., Nom. Bot. ed. 3: 290 (1797). Family: Flacourtiaceae

47. Flacourtia indica (Burm. f.) Merr., Interpr. Rump. Herb. Amb. 377 (1917).

Family: Flacourtiaceae

48. Gynocardia odorata R. Br. in Roxb. Pl. Corom. 3: 95, t. 299 (1820).

Family: Flacourtiaceae

49. Salomonia cantoniensis Lour., Pl. Cochinch. 1:14 (1790). Family: Polygalaceae

50. Securidaca inappendiculata Hassk., Pl. Jav. Rar. 295 (1848). Family: Polygalaceae

51. Xanthophyllum flavescens Roxb., Pl. Corom. 3: 82, t. 284 fig. 2 (1820). Family: Xanthophyllaceae

52. Hypericum japonicum Thunb. ex Murray in Syst. Veg. ed. 14, 702 (1784). Family: Clusiaceae

53. Triadenum breviflorum (Wall. ex Dyer) Kimura in Nakai \& Honda, Nova Fl. Japan 10:79 (1951). Family: Clusiaceae

54. Cratoxylum sumatranum (Jack.) Blume subsp.neriifolium (Kurz) Gog. in Blumea 15: 463 (1967). Family: Clusiaceae

55. Garcinia affinis Wall. ex Pierr. in Fl. Forest. Cochinch. Fosc. 6:16, t. 78C, 79G (1883).

Family: Clusiaceae 
Table 3 contd.

Name of species, Natural Order as in Hook.f. with recorded area and collector's name

57. Garcinia cowa Roxb. Natural Order: Guttiferae Eastern Bengal - Wall. Cat. 4863

58. Garcinia lanceaefolia Roxb. Natural Order: Guttiferae Silhet - Wall. Cat. 4861 A, B

59. Garcinia pedunculata Roxb. Natural Order: Guttiferae Silhet, Rungpore - Wall. Cat. 4860

60. Garcinia morella Desrouss. Natural Order: Guttiferae Eastern Bengal-Wall. Cat. 4868

61. Garcinia paniculata Roxb. Natural Order: Guttiferae Eastern Bengal - Wall. Cat. 4857

62. Garcinia anomala Planch. \&Trian. Natural Order: Guttiferae Eastern Bengal - Wallich \& c.

63. Garcinia Xanthochymus Hook.f. Natural Order: Guttiferae Eastern Bengal - Wall. Cat. 4837

64. Calophyllum polyanthum Wall. Natural Order: Guttiferae Eastern Bengal - Kurz

65. Mesua ferrea Linn. Natural Order: Guttiferae Eastern Bengal - Wall. Cat. 4834

66. Ternstroemia japonica Thunb. Natural Order: Ternstroemiaceae Eastern Bengal - not mentioned

67. Cleyera grandiflora H. f. \& T. Natural Order: Ternstroemiaceae Eastern Bengal - J.D.H. \& T.T.

68. Eurya acuminata DC. Natural Order: Ternstroemiaceae Eastern Bengal - not mention

69. Saurauja Roxburghii Wall. Natural Order: Ternstroemiaceae Chittagong, Silhet - J. H. D. \& T. T.

70. Schima Wallichii Choisy Natural Order: Ternstroemiaceae Chittagong - Wallich \& c.
Current nomenclature with loc. cite., and family as Cronquist (1981)

56. Garcinia cowa Roxb. ex DC., Prodr. 1:501 (1824). Family: Clusiaceae

57. Garcinia lanceaefolia Roxb., Fl. Ind. 2:623 (1832). Family: Clusiaceae

58. Garcinia pedunculata Roxb. ex Buch-Ham. in Brewster, Edinburgh J. Sci. 7:45. t, 1 (1827).

Family: Clusiaceae

59. Garcinia morella (Gaertn.) Desr. in Lam., Encycl. 3(2): 701, t. 405, F. 4 (1792). Family: Clusiaceae

60. Garcinia sopsopia (Buch-Ham.) Mabberley in Taxon 26: 529 (1977). Family: Clusiaceae

61. Garcinia anomala Planch. \& Triana in Ann. Soc. Nat. Ser. 4, 14: 329 (1860). Family: Clusiaceae

62. Garcinia xanthochymus Hook.f. ex T. Anders. in Hook.f., Fl. Brit. Ind. 1: 269 (1874).

Family: Clusiaceae

63. Calophyllum polyanthum Wall. ex Choisy, Deser. Guttif. Inde.: 43 (1849). Family: Clusiaceae

64. Mesua ferrea L., Sp. Pl. 515 (1753). Family: Clusiaceae

65. Ternstroemia gymnanthera (Wight \& Arn.) Bedd., Fl. Sylv. 91. Pl. 91 (1871).

Family: Theaceae

66. Cleyera japonica Thunb., Nov. Gen. Pl. 68 (1783) var. grandiflora (Wall. ex Choisy) Kobuski. in J. Arn. Arb. 18: 125 (1937). Family: Theaceae

67. Eurya acuminata DC. in Mem. Soc. Phys. Hist. Nat. Geneva 1: 418 (1822). Family: Theaceae

68. Saurauja roxburghii Wall., Pl. As. Rar. 2:40 (1830). Family: Actinidiaceae

69. Schima wallichii (DC.) Korth. in Temminck, Verh. Nat. Gesch. Bot. 143 (1840).

Family: Theaceae 
Table 3 contd.

Name of species, Natural Order as in Hook.f. with recorded area and collector's name

71. Pyrenaria barringtoniaefolia Seem. Natural Order: Ternstroemiaceae Eastern Bengal - Lobb.

72. Camellia caudata Wall. Natural Order: Ternstroemiaceae Silhet - Wallich \& c.

73. Dipterocarpus turbinatus Gaertn. f. Natural Order: Dipterocarpeae Chittagong - Wall. Cat. 952

74. Dipterocarpus pilosus Roxb. Natural Order: Dipterocarpeae Chittagong - Roxburgh \& c.

75. Dipterocarpus scaber Ham. Natural Order: Dipterocarpeae Eastern Bengal - Hamilton

76. Dipterocarpus tuberculatus Roxb. Natural Order: Dipterocarpeae Chittagong - Roxburgh

77. Dipterocarpus alatus Roxb. Natural Order: Dipterocarpeae Chittagong - Wall. Cat. 953

78. Dipterocarpus incanus Roxb. Natural Order: Dipterocarpeae Chittagong - Roxburgh

79. Dipterocarpus costatus Roxb. Natural Order: Dipterocarpeae Chittagong - Not mentined

80. Ancistrocladus Wallichii Planch. Natural Order: Dipterocarpeae Chittagong,Silhet - H f. \& T., De Silva,

81. Vatica scaphula Dyer. Natural Order: Dipterocarpeae Chittagong - Roxb.

82. Vatica lanceaefolia Blume Natural Order: Dipterocarpeae Silhet - Wallich

83. Shorea robusta Gaertn. f. Natural Order: Dipterocarpeae Eastern districts - Wall. Cat. 965

84. Abutilon avicennae Gaertn. Natural Order: Malvaceae Bengal - not mentioned
Current nomenclature with loc. cite., and family as Cronquist (1981)

70. Pyrenaria barringtonifolia (Griff.) Seem., Bonplandia 7: 49 (1859). Family: Theaceae

71. Camellia caudata Wall., [Cat. 27, n. 978 (1829), nom. nud.] Pl. As. Rar. 3: 36 (1830).

Family: Theaceae

72. Dipterocarpus turbinatus Gaertn., De Fruct. 3: 51, t. 188, f. 1 (1805). Family: Dipterocarpaceae

73. Dipterocarpus gracilis Blume, Bijidn. 5: 224 (1825). Family: Dipterocarpaceae

74. Dipterocarpus costatus Gaertn., De. Fruct. 3:50, t. 187 (1805). Family: Dipterocarpaceae

75. Dipterocarpus tuberculatus Roxb., Fl. Ind. 2:614 (1832). Family: Dipterocarpaceae

76. Dipterocarpus alatus Roxb. ex G. Don in Gen. Syst. 1: 813 (1831). Family: Dipterocarpaceae

76. Dipterocarpus alatus Roxb. ex G. Don, Gen. Syst. 1: 813 (1831). Family: Dipterocarpaceae

74. Dipterocarpus costatus Gaertn., De. Fruct. 3: 50, t. 187 (1805). Family: Dipterocarpaceae

77. Ancistrocladus wallichii Planch. in Ann. Sc. Nat. Bot. Ser. 3, 13: 319 (1849).

Family: Ancistrocladaceae

78. Anisoptera scaphula (Roxb.) Pierre. in Fl. For. Cochin., Sub. t. 235, ff. B 13-17 (1888-1891). Family: Dipterocarpaceae

79. Vatica lanceifolia (Roxb.) Blume in Mus. Bot. 2: 31(1852). Family: Dipterocarpaceae

80. Shorea robusta Roxb. ex Gaertn. f., De. Fruct. 3: 48 (1805). Family: Dipterocarpaceae

81. Abutilon theophrasti Medik., Malv.: 28 (1787). Family: Malvaceae 
Table 3 contd.

Name of species, Natural Order as in Hook.f. with recorded area and collector's name

85. Hibiscus surattensis Linn. Natural Order: Malvaceae Bengal - not mentioned

86. Hibiscus fragrans Roxb. Natural Order: Malvaceae Silhet - Roxburgh

87. Hibiscus scandens Roxb. Natural Order: Malvaceae Chittagong - Wall. Cat. 1910, 1910B

88. Hibiscus macrophyllus Roxb. Natural Order: Malvaceae Chittagong, Silhet - Wall. Cat. 1903

89. Hibiscus monihot L. Natural Order: Malvaceae Naturalized in Bengal

90. Hibiscus tiliaceus L. Natural Order: Malvaceae Bengal - not mentioned

91. Thespesia populnea Corr. Natural Order: Malvaceae Bengal - not mentioned

92. Gossypium herbaceum L. Natural Order: Malvaceae Dacca - Wall. Cat.1880

93. Sterculia villosa Roxb. Natural Order: Sterculiaceae Bengal

94. Sterculia Roxburghii Wall. Natural Order: Sterculiaceae Silhet - Wall. Cat. 1124

95. Sterculia colorata Roxb. Natural Order: Sterculiaceae Eastern Bengal - Wall. Cat. 1119 F

96. Sterculia parviflora Roxb. Natural Order: Sterculiaceae Silhet - Wallich

97. Sterculia alata Roxb. Natural Order: Sterculiaceae Chittagong and Silhet - Roxburgh

98. Helicteres elongata Wall. Natural Order: Sterculiaceae Eastern Bengal - J.D.H.
Current nomenclature with loc. cite., and family as Cronquist (1981)

82. Hibiscus surattensis L., Sp. Pl.: 696 (1753). Family: Malvaceae

83. Hibiscus fragrans Roxb., Hort. Beng.: 97 (1814). Family: Malvaceae

84. Hibiscus scandens Roxb., [Hort. Beng.: 57 (1814) nom. nud.] Fl. Ind. ed. Carey 3: 200 (1832). Family: Malvaceae

85. Hibiscus macrophyllus Roxb. ex Hornem., Hort. Hafn. Suppl.: 149 (1819). Family: Malvaceae

86. Abelmoschus monihot (L.) Medik., Malv.: 46 (1787). Paul \& Nayar in Nayar et. al. (eds.) Fasc. Fl. Ind. 19: 74 (1988). Family: Malvaceae

87. Hibiscus tiliaceus L., Sp. Pl.: 694 (1753). Family: Malvaceae

88. Thespesia populnea (L.) Sol. ex Corr. in Ann. Mus. Hist. Nat. Paris 9: 290, t. 8, f. 1 (1807). Family: Malvaceae

89. Gossypium herbaceum L., Sp. Pl.: 693 (1753). Family: Malvaceae

90. Sterculia villosa Roxb., [ H. Beng. 50(1814), nom. nud.], ex Smith in Rees, Cyclop. 34: No. 16 (1816). Family: Sterculiaceae

91. Sterculia roxburghii Wall. in Fl. Asait. Rar. 3; t. 262 (1832). Family: Sterculiaceae

92. Firmiana colorata (Roxb.) R. Br. in Benn. \& Brown., Fl. Java Rar.; 235 (1844).

Family: Sterculiaceae

93. Sterculia parviflora Roxb. ex G. Don in Gen. Hist. 1: 516 (1831). Family: Sterculiaceae

94. Pterygota alata (Roxb.) R. Br. in Benn. Pl. Java Rar. 234 (1844). Family: Sterculiaceae

95. Helicteres elongata Wall., [Cat. No. 1845 (1831), nom. nud.] ex Masters in Hook.f., Fl. Brit. Ind. 1: 365 (1874). Family: Sterculiaceae 
Table 3 contd.

\begin{tabular}{|c|c|}
\hline $\begin{array}{l}\text { Name of species, Natural Order as in Hook.f. } \\
\text { with recorded area and collector's name }\end{array}$ & $\begin{array}{l}\text { Current nomenclature with loc. cite., and } \\
\text { family as Cronquist (1981) }\end{array}$ \\
\hline $\begin{array}{l}\text { 99. Heritiera fomes Buch. } \\
\text { Natural Order: Sterculiaceae } \\
\text { Gangetic Delta, extending inland to Silhet - } \\
\text { not mentioned }\end{array}$ & $\begin{array}{l}\text { 96. Heritiera fomes Buch-Ham. in Symes, An. } \\
\text { Account of an Embassy to the Kingdom of Ava, } \\
\text { ed. 2, 3: } 319 \text { (1800). Family: Sterculiaceae }\end{array}$ \\
\hline $\begin{array}{l}\text { 100. Pterospermum acerifolium Willd } \\
\text { Natural Order: Sterculiaceae } \\
\text { Chittagong - Wall. Cat. } 1170\end{array}$ & $\begin{array}{l}\text { 97. Pterospermum acerifolium (L.) Willd., Sp. Pl. 3: } \\
729 \text { (1800). Family: Sterculiaceae }\end{array}$ \\
\hline $\begin{array}{l}\text { 101. Pterospermum semisagittatum Ham. } \\
\text { Natural Order: Sterculiaceae } \\
\text { Chittagong - H.f. \& T. }\end{array}$ & $\begin{array}{l}\text { 98. Pterospermum semisagittatum Buch-Ham ex } \\
\text { Roxb., Fl. Ind. 3: } 160 \text { (1832). } \\
\text { Family: Sterculiaceae }\end{array}$ \\
\hline $\begin{array}{l}\text { 102. Pterospermum lanceaefolium Roxb. } \\
\text { Natural Order: Sterculiaceae } \\
\text { Silhet - Roxburgh }\end{array}$ & $\begin{array}{l}\text { 99. Pterospermum lanceaefolium Roxb., [Hort. } \\
\text { Beng.: } 50 \text { (1814) nom. nud.] Fl. Ind. 3: } 163 \\
\text { (1832). Family: Sterculiaceae }\end{array}$ \\
\hline $\begin{array}{l}\text { 103. Buettneria aspera Colebrooke } \\
\text { Natural Order: Sterculiaceae } \\
\text { Silhet - De Silva }\end{array}$ & $\begin{array}{l}\text { 100. Byttneria grandiflora DC., Prodr.1: } 486 \\
\text { (Jan.1824). Family: Starculiaceae }\end{array}$ \\
\hline $\begin{array}{l}\text { 104. Buettneria pilosa Roxb. } \\
\text { Natural Order: Sterculiaceae } \\
\text { Chittagong, Silhet - H.f. \& T., De Silva }\end{array}$ & $\begin{array}{l}\text { 101. Byttneria pilosa Roxb., Fl. Ind. 2: 681(1832). } \\
\text { Family: Starculiaceae }\end{array}$ \\
\hline $\begin{array}{l}\text { 105. Brownlowia elata Roxb. } \\
\text { Natural Order: Tiliaceae } \\
\text { Chittagong - Roxburgh, Griffith \& c. }\end{array}$ & $\begin{array}{l}\text { 102. Brownlowia elata Roxb., Pl. Corom. 3: } 61 \\
\text { (1819). Family: Tiliaceae }\end{array}$ \\
\hline $\begin{array}{l}\text { 106. Brownlowia lanceolata Benth. } \\
\text { Natural Order: Tiliaceae } \\
\text { Sunderbunds - Griffith }\end{array}$ & $\begin{array}{l}\text { 103. Brownlowia tersa (L.) Kosterm. in Penerbitan } \\
\text { Majd. Pengetahuan Indonesia 1: } 73 \text { (1995). } \\
\text { Family: Tiliaceae }\end{array}$ \\
\hline $\begin{array}{l}\text { 107. Grewia excelsa Vahl } \\
\text { Natural Order: Tiliaceae } \\
\text { Chittagong - Wall. Cat. } 6307 \text { A }\end{array}$ & $\begin{array}{l}\text { 104. Grewia excelsa Vahl in Symb. B. } 35 \text { (1790). } \\
\text { Family: Tiliaceae }\end{array}$ \\
\hline $\begin{array}{l}\text { 108. Grewia asiatica L. } \\
\text { Natural Order: Tiliaceae } \\
\text { Eastern Bengal - Wall. Cat. } 1089\end{array}$ & $\begin{array}{l}\text { 105. Grewia asiatica L. in Mant. Pl. } 122 \text { (1767). } \\
\text { Family: Tiliaceae }\end{array}$ \\
\hline $\begin{array}{l}\text { 109. Grewia scabrophylla Roxb. } \\
\text { Natural Order: Tiliaceae } \\
\text { Chittagong - Wallich \& C. }\end{array}$ & $\begin{array}{l}\text { 106. Grewia sclerophylla Roxb. ex G. Don in Gen. } \\
\text { Hist. 1: } 550 \text { (1831). Family: Tiliaceae }\end{array}$ \\
\hline $\begin{array}{l}\text { 110. Grewia multiflora Juss. } \\
\text { Natural Order: Tiliaceae } \\
\text { Eastern Bengal - not mentioned }\end{array}$ & $\begin{array}{l}\text { 107. Grewia serrulata DC., Prodr. 1: } 510 \text { (1824). } \\
\text { Family: Tiliaceae }\end{array}$ \\
\hline $\begin{array}{l}\text { 111. Grewia microcos L. } \\
\text { Natural Order: Tiliaceae } \\
\text { Chittagong - Wall. Cat. } 1098\end{array}$ & $\begin{array}{l}\text { 108. Grewia nervosa (Lour.) Panigr., Taxon 34: } 702 \\
\text { (1985). Family: Tiliaceae }\end{array}$ \\
\hline $\begin{array}{l}\text { 112. Triumfetta tomentosa Bojer } \\
\text { Natural Order: Tiliaceae } \\
\text { Silhet - Wallich }\end{array}$ & $\begin{array}{l}\text { 109. Triumfetta pilosa Roth. in Nov. Pl. Sp.: } 223 \\
\text { (1821). Family: Tiliaceae }\end{array}$ \\
\hline
\end{tabular}


Table 3 contd.

Name of species, Natural Order as in Hook.f. with recorded area and collector's name

113. Triumfetta cana Blume Natural Order: Tiliaceae Chittagong - Hook. \& Thoms.

114. Elaeocarpus ganitrus Roxb. Natural Order: Tiliaceae Chittagong - Wallich

115. Elaeocarpus floribundus Blume Natural Order: Tiliaceae Chittagong - Griffith

116. Elaeocarpus serratus L. Natural Order: Tiliaceae Eastern Bengal - Wall. Cat. 2666

117. Elaeocarpus robustus Roxb. Natural Order: Tiliaceae Chittagong - Wall. Cat. 2664 A?

118. Elaeocarpus cuneatus Wight Natural Order: Tiliaceae Chittagong, Silhet - Wight, Beddome

119. Elaeocarpus lanceaefolius Roxb. Natural Order: Tiliaceae Silhet - Wallich, Griffith \& c.

120. Elaeocarpus lucidus Roxb. Natural Order: Tiliaceae Chittagong - Roxburgh, Griffith

121. Elaeocarpus aristatus Roxb. Natural Order: Tiliaceae Silhet - Wallich

122. Elaeocarpus rugosus Roxb. Natural Order: Tiliaceae Chittagong - Roxburgh

123. Elaeocarpus acuminatus Wall. Natural Order: Tiliaceae Silhet - Wallich \& c.

124. Elaeocarpus prunifolius Wall. Natural Order: Tiliaceae Silhet - Wallich

125. Elaeocarpus Varunua Ham. Natural Order: Tiliaceae Chittagong,Silhet - Wall. Cat. 2666 G,H

126. Elaeocarpus integra Wall. Natural Order: Tiliaceae Silhet - Wallich
Current nomenclature with loc. cite., and family as Cronquist (1981)

110. Triumfetta oblique Roth. in Nov. Pl. Sp. 224 (1821). Family: Tiliaceae

111. Elaeocarpus angustifolius Blume in Bijdr. Fl. Ned. Ind. 3: 120 (1825). Family: Elaeocarpaceae

112. Elaeocarpus floribundus Blume in Bijdr.: 120 (1825). Family: Elaeocarpaceae

113. Elaeocarpus serratus L., Sp. Pl.: 515 (1753). Family: Elaeocarpaceae

114. Elaeocarpus tectorius (Lour.) Poir. in Lam., Encycl. Suppl. 2: 704 (1812).

Family: Elaeocarpaceae

113. Elaeocarpus serratus L., Sp. Pl.: 515 (1753). Family: Elaeocarpaceae

115. Elaeocarpus lanceifolius Roxb., Fl. Ind. 2: 598 (1832). Family: Elaeocarpaceae

116. Elaeocarpus lucidus Roxb., Fl. Ind. 2: 600 (1832). Family: Elaeocarpaceae

117. Elaeocarpus aristatus Roxb., Fl. Ind. 2: 599 (1832). Family: Elaeocarpaceae

118. Elaeocarpus rugosus Roxb. ex G. Don in Gen Hist. 1: 559 (1831). Family: Elaeocarpaceae

119. Elaeocarpus acuminatus Wall. ex Masters in Hook.f., Fl. Brit. Ind. 1: 406 (1874).

Family: Elaeocarpaceae

120. Eleocarpus prunifolius (C. Muell.) Masters in Hook.f., Fl. Brit. Ind. 1: 407 (1874).

Family: Elaeocarpaceae

121. Elaeocarpus varunua Buch-Ham. ex Masters in Hook.f., Fl. Brit. Ind. 1: 407 (1874).

Family: Elaeocarpaceae

122. Elaeocarpus petiolatus (Jack.) Wall. ex Steud. in Nom. Bot. ed. 1: 545 (1840).

Family: Elaeocarpaceae 
Table 3 contd.

Name of species, Natural Order as in Hook.f. with recorded area and collector's name

127. Reinwardtia trigyna Planch. Natural Order: Lineae Chittagong - Wall. Cat. 1505

128. Erythroxylon Kunthianum Wall. Natural Order: Lineae Eastern Bengal - Wall. Cat. 6849

129. Aspidopterys nutans Hook.f. Natural Order: Malpighiaceae Chittagong - Roxburgh \& c.

130. Aspidopterys natuns var. rotundifolia A. Juss. Natural Order: Malpighiaceae Chittagong - Roxburgh

131. Impatiens flavida H. f. \& T. Natural Order: Geraniaceae Silhet - Wallich \& c.

132. Impatiens tripetala Roxb. Natural Order: Geraniaceae Silhet - Wall. Cat. 4742

133. Hydrocera triflora W. \& A. Natural Order: Geraniaceae Throughout Bengal

134. Zanthoxylum Budrunga Wall. Natural Order: Rutaceae Chittagong, Silhet - Wall. Cat. 1211

135. Acronychia laurifolia Blume Natural Order: Rutaceae Chittagong - Wall. Cat. 1205

136. Micromelum pubecens Blume Natural Order: Rutaceae Chittagong - Wall. Cat. 6371

137. Murraya exotica Linn. Natural Order: Rutaceae Chittagong - Wall. Cat. 6368

138. Murraya koenigii Spreng. Natural Order: Rutaceae Bengal - not mentioned

139. Clausena heptaphylla W. \& A. Natural Order: Rutaceae Chittagong, Silhet - H. f. \&T., Wallich

140. Clausena excavata Burm. Natural Order: Rutaceae Chittagong, Silhet - Wallich \& c.
Current nomenclature with loc. cite., and family as Cronquist (1981)

123. Reinwardtia indica Dumort., Com. Bot.: 19 (1822). Family: Linaceae

124. Erythroxylum kunthianum Wall. ex Kurz in J. Asiat. Soc. Beng. 41: 294 (1872).

Family: Erythroxylaceae

125. Aspidopterys nutans (Roxb. ex DC.) A. Juss. in Ann. Sc. Nat. 2. Ser. Bot. 5, 13: 267 (1840).

Family: Malpighiaceae

126. Aspidopterys orbiculata (Roxb. ex Wall.) Niedenzu in Arb. Bot. Inst. Kerigl. Lyceums Hosianum Braunsberg 6: 14 (1915).

Family: Malpighiaceae

127. Impetiens trilobata Colebr. in Hook.f., Exot. Fl. 2:f. 141 (1825). Family: Balsaminaceae

128. Impetiens tripetala Roxb. ex DC., Prodr. 1: 687 (1824). Family: Balsaminaceae

129. Hydrocera triflora (L.) Wight \& Arn., Prod. 140 (1834). Family: Balsaminaceae

130. Zanthoxylum rhetsa (Roxb.) DC., Prodr. 1: 728 (1824). Family: Rutaceae

131. Acronychia padunculata (L.) Miq. In Fl. Ind. Bot. Suppl. 532 (1861). Family: Rutaceae

132. Micromelum minutum (G. Froster) Wight \& Arn., Prodr. Fl. Ind. Orient.: 448, 468 (1834).

Family: Rutaceae

133. Murraya paniculata (L.) Jack. in Malayan Misc. 1(5): 31 (1820). Family: Rutaceae

134. Murraya koenigii (L.) Spreng., Syst. Veg. 2: 315 (1826). Family: Rutaceae

135. Clausena heptaphylla (Roxb.) Wight \& Arn. ex Steud., Prodr. Fl. Ind. Or. 95 in nota (1834). Family: Rutaceae

136. Clausena excavata Burm. f., Fl. Ind. 87. t. 29. f. 2 (1768). Family: Rutaceae 
Table 3 contd.

Name of species, Natural Order as in Hook.f. with recorded area and collector's name

141. Clausena wampi Blanco Natural Order: Rutaceae Eastern Islands

142. Clausena suffruticosa W. \& A. Natural Order: Rutaceae Chittagong - Roxburgh

143. Luvunga scandens Ham. Natural Order: Rutaceae Eastern Bengal - Wall. Cat. 6382

144. Paramignya griffithii Hook. f. Natural Order: Rutaceae Silhet - Wallich

145. Paramignya citrifolia Hook.f. Natural Order: Rutaceae Chittagong - Roxburgh

146. Atalantia monophylla Correa Natural Order: Rutaceae Silhet - H f. \& T.

147. Citrus medica Linn. Natural Order: Rutaceae Chittagong - H. f. \& T.

148. Brucea mollis Wall. Natural Order: Simarubeae Silhet - Wallich

149. Ochna aquarrosa Linn. Natural Order: Ochnaceae Silhet - Wall. Cat. 2805

150. Balsamodendron Roxburghii Arn. Natural Order: Burseraceae Silhet - Roxburgh \& c.

151. Bursera serrata Colebr. Natural Order: Burseraceae Chittagong - Wall. Cat. 8492

152. Canarium bengalense Roxb. Natural Order: Burseraceae Silhet - Roxburgh

153. Dysoxylum grande Hiern Natural Order: Meliaceae Silhet - (Wall.)

154. Dysoxylum Hamiltonii Hiern Natural Order: Meliaceae Silhet - Wall.Cat. 4882
Current nomenclature with loc. cite., and family as Cronquist (1981)

137. Clausena lansium (Lour.) Skeels in U. S. D. A. Bur. Pl. Industr. Bull. 168: 31(1909).

Family: Rutaceae

138. Clausena suffruticosa (Roxb.) Wight \& Arn., Prodr.: 96 (1834). Family: Rutaceae

139. Luvunga scandens (Roxb.) Buch-Ham. ex Wight in Wight \& Arn., Prodr. 90 (1834).

Family: Rutaceae

140. Paramignya scandens (Griff.) Craib in Fl. Siam. Enum. 1: 235 (1926). Family: Rutaceae

140. Paramignya scandens (Griff.) Craib in Fl. Siam. Enum. 1: 235 (1926). Family: Rutaceae

141. Atalantia monophylla (L.) DC., Prodr. 1: 535 (1824). Family: Rutaceae

142. Citrus medica L., Sp. Pl. 782 (1753). Family: Rutaceae

143. Brucea mollis Wall. ex Kurz in J. As. S. Beng. 42: 64 (1873). Family: Simaroubaceae

144. Ochna squarrosa L., Sp. Pl.: 732 (1753). Family: Ochnaceae

145. Cammiphora agallocha (Wight \& Arn.) Engl. in DC., Mongr. Phan. 4: 11 (1883).

Family: Burseraceae

146. Protium serratum (Wall. ex Colebr.) Engl. in DC., Monogr. Phan. 4: 88 (1883).

Family: Burseraceae

147. Canarium bengalense Roxb., Fl. Ind. 3: 316 (1832). Family: Burseraceae

148. Dysoxylum grande Hiern. in Hook.f., Fl. Brit. Ind. 1: 547 (1875). Family: Meliaceae

149. Dysoxylum mollissimum Blume, Bijdr. 175 (1825). Family: Meliaceae 
Table 3 contd.

\begin{tabular}{|c|c|}
\hline $\begin{array}{l}\text { Name of species, Natural Order as in Hook.f. } \\
\text { with recorded area and collector's name }\end{array}$ & $\begin{array}{l}\text { Current nomenclature with loc. cite., and } \\
\text { family as Cronquist (1981) }\end{array}$ \\
\hline $\begin{array}{l}\text { 155. Aglaia Wallichii Hiern } \\
\text { Natural Order: Meliaceae } \\
\text { Silhet - Wallich, Griffith }\end{array}$ & $\begin{array}{l}\text { 150. Aglaia wallichii Hiern. in Hook.f., Fl. Brit. Ind. 1: } \\
555 \text { (1875). Family: Meliaceae }\end{array}$ \\
\hline $\begin{array}{l}\text { 156. Aglaia edulis A. Gray. } \\
\text { Natural Order: Meliaceae } \\
\text { Silhet - Wallich, J. D. H. \& T. T. }\end{array}$ & $\begin{array}{l}\text { 151. Aglaia edulis (Roxb.) Wall., Calc. Gar. Rep. } 26 \\
\text { (1840). Family: Miliaceae }\end{array}$ \\
\hline $\begin{array}{l}\text { 157. Amoora Rohituka W. \& A. } \\
\text { Natural Order: Meliaceae } \\
\text { Silhet - Wall. Cat. } 4888\end{array}$ & $\begin{array}{l}\text { 152. Aphanamixis polystachya (Wall.) R. N. Parker, } \\
\text { Ind. For. 57: } 486 \text { (1931). Family: Meliaceae }\end{array}$ \\
\hline $\begin{array}{l}\text { 158. Amoora chittagonga Hiern. } \\
\text { Natural Order: Meliaceae } \\
\text { Chittagong - J. D. H. \& T. T. }\end{array}$ & $\begin{array}{l}\text { 153. Aglaia chittagonga Miq. in Ann. Mus. Bot. } \\
\text { Lugduno-Batavum 4:44 (1868). } \\
\text { Family: Meliaceae }\end{array}$ \\
\hline $\begin{array}{l}\text { 159. Amoora cucullata Roxb. } \\
\text { Natural Order: Meliaceae } \\
\text { Sunderbunds - Wallich }\end{array}$ & $\begin{array}{l}\text { 154. Aglaia cucullata (Roxb.) Pellegr. in Fl. Indo- } \\
\text { Chine. 1: } 771 \text { (1911). Family: Meliaceae }\end{array}$ \\
\hline $\begin{array}{l}\text { 160. Walsura robusta Roxb. } \\
\text { Natural Order: Meliaceae } \\
\text { Silhet - Wallich }\end{array}$ & $\begin{array}{l}\text { 155. Walsura robusta Roxb., Fl. Ind. 2:386 (1832). } \\
\text { Family: Meliaceae }\end{array}$ \\
\hline $\begin{array}{l}\text { 161. Heynea trijuga Roxb. } \\
\text { Natural Order: Meliaceae } \\
\text { Bengal - not mentioned }\end{array}$ & $\begin{array}{l}\text { 156. Heynea trijuga Sims in Curtis, Bot. Mag. 41: t. } \\
1738 \text { (1815). Family: Meliaceae }\end{array}$ \\
\hline $\begin{array}{l}\text { 162. Chailletia gelonioides Hook.f. } \\
\text { Natural Order: Chailletiaceae } \\
\text { Chittagong, Silhet - Wall. Cat. } 4342\end{array}$ & $\begin{array}{l}\text { 157. Dichapetalum gelonioides (Roxb.) Engl. in Engl. } \\
\text { \& Prantl., Pflanzenfam. 3, 4: } 348 \text { (1896). } \\
\text { Family: Dichapetalaceae }\end{array}$ \\
\hline $\begin{array}{l}\text { 163. Olax imbricata Roxb. } \\
\text { Natural Order: Olacineae } \\
\text { Chittagong - Wallich }\end{array}$ & $\begin{array}{l}\text { 158. Olax imbricata Roxb., Fl. Ind. 1: } 169 \text { (1820). } \\
\text { Family: Olacaceae }\end{array}$ \\
\hline $\begin{array}{l}\text { 164. Olax acuminata Wall. } \\
\text { Natural Order: Olacineae } \\
\text { Silhet - Wallich }\end{array}$ & $\begin{array}{l}\text { 159. Olax acuminata Wall. ex Benth. in Trans. Linn. } \\
\text { Soc. 18: } 678 \text { (1841). Family: Olacaceae }\end{array}$ \\
\hline $\begin{array}{l}\text { 165. Erythropalum scandens Blume } \\
\text { Natural Order: Olacineae } \\
\text { Silhet - Wallich }\end{array}$ & $\begin{array}{l}\text { 160. Erythropalum scandens Blume, Bijdr.: } 922 \\
\text { (1826). Family: Olacaceae }\end{array}$ \\
\hline $\begin{array}{l}\text { 166. Schoepfia acuminata Wall. } \\
\text { Natural Order: Olacineae } \\
\text { Silhet - Wallich }\end{array}$ & $\begin{array}{l}\text { 161. Schoepfia fragrans Wall. in Roxb. Fl. Ind. ed. 2: } \\
188 \text { (1832). Family: Olacaceae }\end{array}$ \\
\hline $\begin{array}{l}\text { 167. Lepionurus oblongifolius Mast. } \\
\text { Natural Order: Olacineae } \\
\text { Eastern Bengal - Wall. Cat. 7206F }\end{array}$ & $\begin{array}{l}\text { 162. Lepionurus sylvestris Blume, Bijdr. } 1148 \text { (1826). } \\
\text { Family: Opiliaceae }\end{array}$ \\
\hline $\begin{array}{l}\text { 168. Gomphandra axillaris Wall. } \\
\text { Natural Order: Olacineae } \\
\text { Silhet - Wallich }\end{array}$ & $\begin{array}{l}\text { 163. Gomphandra tetrandra (Wall. ex Roxb.) } \\
\text { Sleum., Notizbl. Berlin-Dablen 15: } 238 \text { (1940). } \\
\text { Family: Icacinaceae }\end{array}$ \\
\hline
\end{tabular}


Table 3 contd.

Name of species, Natural Order as in Hook.f. with recorded area and collector's name

169. Natsiatum herpeticum Ham. Natural Order: Olacineae Chittagong, Silhet - Wall. Cat. 4252

170. Iodes Hookeriana Baill. Natural Order: Olacineae Chittagong - H. f. \& T.

171. Iodes Thomsoniana Baill. Natural Order: Olacineae Chittagong - H.f. \& T.

172. Cardiopteris lobata $\mathrm{R}$. Br. Natural Order: Olacineae Silhet - Wall.Cat.8033

173. Ilex Griffithii Hook.f. Natural Order: Ilicineae Silhet - H. f. \& T. T.

174. Ilex Godajam Colebr. Natural Order: Ilicineae Silhet - Wall. Cat. 4329

175. Euonymus glaber Roxb. Natural Order: Celastrineae East Bengal - Griffith

176. Euonymus cinereus Laws. Natural Order: Celastrineae East Bengal - Griffith

177. Euonymus pendulus Wall. Natural Order: Celastrineae East Bengal - Griffith

178. Lophopetalum fimbriatum Wight Natural Order: Celastrineae Silhet - Wallich

179 Celastrus venulosa Wall. Natural Order: Celastrineae Silhet - Wallich

180. Celastrus monosperma Roxb. Natural Order: Celastrineae Eastern Bengal-Wallich, Griffith

181. Gymnosporia neglecta Wall. Natural Order: Celastrineae Silhet - Wallich

182. Gymnosporia Wallichiana Spreng. Natural Order: Celastrineae Eastern Bengal - Herb. Griffith
Current nomenclature with loc. cite., and family as Cronquist (1981)

164. Natsiatum herpaticum Buch-Ham. ex Arn. in Edinb. New Philos. J. 16: 314 (1834).

Family: Icacinaceae

165. Iodes hookeriana Baill., Adans. 10: 268 (18711872). Family: Icacinaceae

166. Iodes thomsoniana Baill., Adans. 10: 270 (18711872). Family: Icacinaceae

167. Peripterygium quinquelobum Hassk. in Tijd. Nat. Gesch. Phys. 10: 142 (1843). Family: Cardiopteridaceae

168. Ilex triflora Blume, Bijdr.: 1150 (1826). Family: Aquifoliaceae

169. Ilex godajam Colebr. ex Hook.f., Fl. Brit. Ind. 1: 604 (1875). Family: Aquifoliaceae

170. Euonymus glaber Roxb., Fl. Ind. 2: 403 (1824). Family: Celastraceae

171. Euonymus cinereus Laws. in Hook.f., Fl. Brit. Ind. 1: 611 (1875). Family: Celastraceae

172. Euonymus pendulus Wall. in Roxb., Fl. Ind. 2: 406 (1824). Family: Celastraceae

173. Lephopetalum wightianum Arn. in Ann. Mag. Nat. Hist. 3: 151(1839).

Family: Celastraceae

174. Celastrus venulosa Wall. ex Hook.f. \& Thom. in Fl. Brit. Ind. 1: 618 (1872).

Family: Celastraceae

175. Celastrus monospermus Roxb., Fl. Ind. 2: 394 (1824). Family: Celastraceae

176. Celastrus stylosus Wall. ex Roxb., Fl. Ind. 2: 401 (1824). Family: Celastraceae

177. Maytenus wallichii (G. Don) Bennet. \& Sahni., Ind. For. 103(6): 387 (1977).

Family: Celastraceae 
Table 3 contd.

\begin{tabular}{|c|c|}
\hline $\begin{array}{l}\text { Name of species, Natural Order as in Hook.f. } \\
\text { with recorded area and collector's name }\end{array}$ & $\begin{array}{l}\text { Current nomenclature with loc. cite., and } \\
\text { family as Cronquist (1981) }\end{array}$ \\
\hline $\begin{array}{l}\text { 183. Kurrimia pulcherrima Wall. } \\
\text { Natural Order: Celastrineae } \\
\text { Chittagong, Silhet - Wall. Cat. } 4334\end{array}$ & $\begin{array}{l}\text { 178. Bhesa robusta (Roxb.) Ding Hou in Blume } \\
\text { Suppl. 4: } 152 \text { (1958). Family: Celastraceae }\end{array}$ \\
\hline $\begin{array}{l}\text { 184. Hippocratea obtusifolia Roxb. } \\
\text { Natural Order: Celastrineae } \\
\text { Silhet - Wallich }\end{array}$ & $\begin{array}{l}\text { 179. Loeseneriella africana (Willd.) Wilczek in Fl. } \\
\text { Cong. Belge. \& Ruanda-Brundi 9: } 154 \text { (1960). } \\
\text { Family: Hippocrateaceae }\end{array}$ \\
\hline $\begin{array}{l}\text { 185. Hippocratea Grahami Wight } \\
\text { Natural Order: Celastrineae } \\
\text { Silhet - Wallich }\end{array}$ & $\begin{array}{l}\text { 180. Hippocratea grahamii Wight in Illustr. Ind. Bot. } \\
\text { 1: } 130 \text { (1840). Family: Hippocrateaceae }\end{array}$ \\
\hline $\begin{array}{l}\text { 186. Salacia prinoides DC. } \\
\text { Natural Order: Celastrineae } \\
\text { Silhet - H. f. \& T. }\end{array}$ & $\begin{array}{l}\text { 181. Salacia chinensis L., Mant. ed. 2: } 293 \text { (1771). } \\
\text { Family: Hippocrateaceae }\end{array}$ \\
\hline $\begin{array}{l}\text { 187. Salacia floribunda Wight } \\
\text { Natural Order: Celastrineae } \\
\text { Silhet - De Silva }\end{array}$ & $\begin{array}{l}\text { 182. Salacia floribunda Wight in Illustr. Ind. Bot. 1: } \\
134 \text { (1840). Family: Hippocrateaceae }\end{array}$ \\
\hline $\begin{array}{l}\text { 188. Ventilago calyculata Tulasne } \\
\text { Natural Order: Rhamneae } \\
\text { Silhet - Wall. Cat. } 4268 \text { b }\end{array}$ & $\begin{array}{l}\text { 183. Ventilago denticulate Willd., Ges. Natur. Fr. } \\
\text { Neue. Schr. 3: } 417 \text { (1801). Family: Rhamnaceae }\end{array}$ \\
\hline $\begin{array}{l}\text { 189. Zizyphus glabrata Heyne. } \\
\text { Natural Order: Rhamneae } \\
\text { Eastern Bengal - Griffith }\end{array}$ & $\begin{array}{l}\text { 184. Ziziphus glabrata Heyne. ex Roth., Nov. Pl. Sp.: } \\
159 \text { (1821). Family: Rhamnaceae }\end{array}$ \\
\hline $\begin{array}{l}\text { 190. Zizyphus funiculosa Ham. } \\
\text { Natural Order: Rhamneae } \\
\text { Silhet - Wallich \& c. }\end{array}$ & $\begin{array}{l}\text { 185. Ziziphus funiculosa Buch-Ham. ex Lows. in } \\
\text { Hook.f., Fl. Brit. Ind. I: } 636 \text { (1875). } \\
\text { Family: Rhamnaceae }\end{array}$ \\
\hline $\begin{array}{l}\text { 191. Zizyphus rugosa Lamk. } \\
\text { Natural Order: Rhamneae } \\
\text { Silhet - Wallich \& c. }\end{array}$ & $\begin{array}{l}\text { 186. Ziziphus rugosa Lam., Encycl. 3: } 319 \text { (1789). } \\
\text { Family: Rhamnaceae }\end{array}$ \\
\hline $\begin{array}{l}\text { 192. Berchemia floribunda Wall. } \\
\text { Natural Order: Rhamneae } \\
\text { Eastern Bengal - Griffith }\end{array}$ & $\begin{array}{l}\text { 187. Berchemia floribunda (Wall.) Brongn., Mem. } \\
\text { Fam. Rhamn. 50, t. } 2.1 \text { (1826) in Ann. Sci. Nat. } \\
\text { 10: 357, t. 13, 1(1827). Family: Rhamnaceae }\end{array}$ \\
\hline $\begin{array}{l}\text { 193. Vitis pentagona Roxb. } \\
\text { Natural Order: Ampelideae } \\
\text { Chittagong - Roxburgh }\end{array}$ & $\begin{array}{l}\text { 188. Vitis pentagona (Roxb.) Lows. in Hook.f., Fl. } \\
\text { Brit. Ind. 1: } 646 \text { (1875). Family: Vitaceae }\end{array}$ \\
\hline $\begin{array}{l}\text { 194. Vitis repens W. \& A. } \\
\text { Natural Order: Ampelideae } \\
\text { Chittagong, Silhet -Wall. Cat. } 5990\end{array}$ & $\begin{array}{l}\text { 189. Cissus repens Lam. in Encycl. Math. Bot. 1: } 31 \\
\text { (1783). Family: Vitaceae }\end{array}$ \\
\hline $\begin{array}{l}\text { 195. Vitis discolor Dalz. } \\
\text { Natural Order: Ampelideae } \\
\text { Chittagong, Silhet - Wall. Cat. } 6010\end{array}$ & $\begin{array}{l}\text { 190. Cissus javana DC., Prodr. 1: } 628 \text { (1824). } \\
\text { Family: Vitaceae }\end{array}$ \\
\hline $\begin{array}{l}\text { 196. Vitis repanda W. \& A. } \\
\text { Natural Order: Ampelideae } \\
\text { Silhet - Wall. Cat. } 6002\end{array}$ & $\begin{array}{l}\text { 191. Cissus rependa Vahl, Symb. 3: } 18 \text { (1794). } \\
\text { Family: Vitaceae }\end{array}$ \\
\hline
\end{tabular}


Table 3 contd.

Name of species, Natural Order as in Hook.f. with recorded area and collector's name

197. Vitis adnata Wall Natural Order: Ampelideae Silhet - Wall. Cat. 5998

198. Vitis barbata Wall. Natural Order: Ampelideae Silhet - Wall. Cat. 5995 c,d

199. Vitis lanata Roxb. Natural Order: Ampelideae Chittagong - Wall. Cat. 5995 a,b,e

200. Vitis latifolia Roxb. Natural Order: Ampelideae Silhet - Wall. Cat. 5993 ?

201. Vitis montana Laws. Natural Order: Ampelideae Silhet - Hook. f. \& T.

202. Vitis parvifolia Roxb. Natural Order: Ampelideae Eastern Bengal - Roxburgh

203. Vitis bracteolata Wall. Natural Order: Ampelideae Silhet - De Silva

204. Vitis angustifolia Wall. Natural Order: Ampelideae Silhet - Wall. Cat. 6033

205. Vitis elongata Wall. Natural Order: Ampelideae Silhet - Wall. Cat. 6016

206. Vitis auriculata Roxb. Natural Order: Ampelideae Chittagong - H.f. \& T.

207. Vitis capriolata Don Natural Order: Ampelideae Silhet - Roxburgh

208. Vitis mollis Wall. Natural Order: Ampelideae Chittagong, Silhet - Wall. Cat. 6025

209. Vitis dubia Laws. Natural Order: Ampelideae Chittagong? - H. f. \& T.

210. Vitis Hookeri Laws. Natural Order: Ampelideae Chittagong - H. f. \& T.
Current nomenclature with loc. cite., and family as Cronquist (1981)

192. Cissus adnata Roxb., Fl. Ind. ed. Carey 1:405 (1814). Family: Vitaceae

193. Ampelocissus barbata (Wall.) Planch. in DC., Monogr. Phan. 5: 375 (1887). Family: Vitaceae

194. Vitis lanata Roxb., Fl. Ind. 2: 472 (1814). Family: Vitaceae

195. Ampelocissus latifolia (Roxb.) Planch., J. Vigne. Amer.: 374 (1884). Family: Vitaceae

195. Ampelocissus latifolia (Roxb.) Planch., J. Vigne. Amer.: 374 (1884). Family: Vitaceae

196. Vitis parvifolia Roxb., Fl. Ind. 2: 475 (1820). Family: Vitaceae

197. Tetrastigma bracteolatum (Wall.) Planch. In DC., Monogr. Phan. 5: 428 (1887).

Family: Vitaceae

198. Tetrastigma angustifolium (Roxb.) Planch. in DC., Monogr. Phan. 5: 439 (1887).

Family: Vitaceae

199. Cissus elongata Roxb., Fl. Ind. 1: 411 (1832).

Family: Vitaceae

200. Cyphostemma auriculata (Roxb.) Singh \& Shetty in Fl. Bhutan. 2(1): 160 (1991).

Family: Vitaceae

201. Tetrastigma serrulatum (Roxb.) Planch. in DC., Monogr. Phan. 5: 432 (1887).

Family: Vitaceae

202. Cayratia japonica (Thanb.) Gagnep., Nat. Syst. 1: 349 (1911). Family: Vitaceae

203. Tetrastigma dubium (Laws.) Planch. in DC., Monogr. Phan. 5: 437 (1887).

Family: Vitaceae

204. Tetrastigma leucostaphyllum (Dennst.) Alston ex Mabb. in Taxon 20: 539 (1977).

Family: Vitaceae 
Table 3 contd.

\begin{tabular}{|c|c|}
\hline $\begin{array}{l}\text { Name of species, Natural Order as in Hook.f. } \\
\text { with recorded area and collector's name }\end{array}$ & $\begin{array}{l}\text { Current nomenclature with loc. cite., and } \\
\text { family as Cronquist (1981) }\end{array}$ \\
\hline $\begin{array}{l}\text { 211. Vitis pedata Vahl } \\
\text { Natural Order: Ampelideae } \\
\text { Silhet - Wall. Cat. } 6027\end{array}$ & $\begin{array}{l}\text { 205. Cayratia pedata (Lam.) Juss. ex Gagnep., Not. } \\
\text { Syst. 1:346 (1911). } \\
\text { Family: Vitaceae }\end{array}$ \\
\hline $\begin{array}{l}\text { 212. Vitis rubifolia Wall. } \\
\text { Natural Order: Ampelideae } \\
\text { Silhet - Wallich }\end{array}$ & $\begin{array}{l}\text { 206. Vitis rubifolia Wall. in Roxb., Fl. Ind. 2: } 480 \\
\text { (1824). Family: Vitaceae }\end{array}$ \\
\hline $\begin{array}{l}\text { 213. Leea macrophylla Roxb. } \\
\text { Natural Order: Ampelideae } \\
\text { Bengal - Wall. Cat. } 6818\end{array}$ & $\begin{array}{l}\text { 207. Leea macrophylla Roxb. ex Hornem., Hort. } \\
\text { Hafn. 1: } 231 \text { (1813). Family: Leeaceae }\end{array}$ \\
\hline $\begin{array}{l}\text { 214. Leea crispa Willd. } \\
\text { Natural Order: Ampelideae } \\
\text { Chittagong, Silhet - Wallich }\end{array}$ & $\begin{array}{l}\text { 208. Leea crispa L., Syst. Nat. ed. 12, 2: } 627 \text { (1767). } \\
\text { Family: Leeaceae }\end{array}$ \\
\hline $\begin{array}{l}\text { 215. Leea hirta Roxb. } \\
\text { Natural Order: Ampelideae } \\
\text { Chittagong, Silhet - Wall. Cat. } 6822\end{array}$ & $\begin{array}{l}\text { 209. Leea aequata L., Syst. Nat. ed. 12, 2: } 627 \text { (1767). } \\
\text { Family: Leeaceae }\end{array}$ \\
\hline $\begin{array}{l}\text { 216. Allophylus cobbe Blume } \\
\text { Natural Order: Sapindaceae } \\
\text { Silhet - Wall. Cat. } 8066\end{array}$ & $\begin{array}{l}\text { 210. Allophyllus triphyllus (Brum. f.) Merr. in Phil. J. } \\
\text { Sci. 19: } 363 \text { (1921). Family: Sapindaceae }\end{array}$ \\
\hline $\begin{array}{l}\text { 217. Cupania pentapetala W. \& A. } \\
\text { Natural Order: Sapindaceae } \\
\text { Silhet - Roxburgh }\end{array}$ & $\begin{array}{l}\text { 211. Cupania pentapetala Wight \& Arn., Prodr. } 113 \\
\text { (1834). Family: Sapindaceae }\end{array}$ \\
\hline $\begin{array}{l}\text { 218. Cupania sumatrana Miq. } \\
\text { Natural Order: Sapindaceae } \\
\text { Silhet - Gomez, H. f. \& T. }\end{array}$ & $\begin{array}{l}\text { 212. Mischocarpus pentapetalus (Roxb.) Radlk. in } \\
\text { Sapind. Holl.-Ind.: } 43 \text { (1879). } \\
\text { Family: Sapindaceae }\end{array}$ \\
\hline $\begin{array}{l}\text { 219. Sapindus trifoliatus Linn. } \\
\text { Natural Order: Sapindaceae } \\
\text { Bengal - Cultivated }\end{array}$ & $\begin{array}{l}\text { 213. Sapindus emarginatus Vahl, Symb. Bot. 3: } 54 \\
\text { (1794). Family: Sapindaceae }\end{array}$ \\
\hline $\begin{array}{l}\text { 220. Sapindus Mukorossi Gaertn. } \\
\text { Natural Order: Sapindaceae } \\
\text { Silhet }\end{array}$ & $\begin{array}{l}\text { 214. Sapindus saponaria L., Sp. Pl.: } 367 \text { (1753). } \\
\text { Family: Sapindaceae }\end{array}$ \\
\hline $\begin{array}{l}\text { 221. Sapindus attenuatus Wall. } \\
\text { Natural Order: Sapindaceae } \\
\text { Silhet - Wallich, Griffith \& c. }\end{array}$ & $\begin{array}{l}\text { 215. Aphania rubra Radlk. in Sitzb. Math. Phys. } \\
\text { Acad. Mucnch.: } 238 \text { (1878). } \\
\text { Family: Sapindaceae }\end{array}$ \\
\hline $\begin{array}{l}\text { 222. Sapindus Danura Voigt } \\
\text { Natural Order: Sapindaceae } \\
\text { Chittagong, Silhet - Wall. Cat. } 8051\end{array}$ & $\begin{array}{l}\text { 216. Aphania danura (Voigt.) Radlk., in Sitzb. Math. } \\
\text { Phys. Acad. Mucnch. 8: } 238 \text { (1878); } \\
\text { Family: Sapindaceae }\end{array}$ \\
\hline $\begin{array}{l}\text { 223. Xerospermum Noronhianum } \\
\text { Blume. Natural Order: Sapindaceae } \\
\text { Silhet - Wallich }\end{array}$ & $\begin{array}{l}\text { 217. Xerospermum noronhianum (Blume) Blume, } \\
\text { Rumphia 3: } 100 \text { (1847). Family: Sapindaceae }\end{array}$ \\
\hline $\begin{array}{l}\text { 224. Nephelium longana Camb. } \\
\text { Natural Order: Sapindaceae } \\
\text { Eastern Bengal - Wall. Cat. } 8049\end{array}$ & $\begin{array}{l}\text { 218. Dimocarpus longan Lour., Fl. Cochinch.: } 233 \\
\text { (1790). Family: Sapindaceae }\end{array}$ \\
\hline
\end{tabular}


Table 3 contd.

\begin{tabular}{lc}
\hline $\begin{array}{l}\text { Name of species, Natural Order as in Hook.f. } \\
\text { with recorded area and collector's name }\end{array}$ & $\begin{array}{l}\text { Current nomenclature with loc. cite., and } \\
\text { family as Cronquist (1981) }\end{array}$ \\
\hline $\begin{array}{l}\text { 225. Harpullia cupanoides Roxb. } \\
\text { Natural Order: Sapindaceae }\end{array}$ & $\begin{array}{l}\text { 219. Harpullia cupaniodes Roxb., Fl. Ind. ed. Carey } \\
\text { Chittagong - Roxburgh }\end{array}$ \\
$\begin{array}{l}\text { 226. Turpinia pomifera DC. } \\
\text { Natural Order: Sapindaceae }\end{array}$ & $\begin{array}{l}\text { 220. Turpinia pomifera (Roxb.) DC., Prodr. 2: } 3 \\
\text { (1825). Family: Staphyleaceae }\end{array}$ \\
\hline
\end{tabular}

\section{Acknowledgement}

The authors are grateful to the University Grants Commission, Bangladesh for providing fund to carry out this research.

\section{References}

Ahmed, Z.U., Hassan, M.A., Begum, Z.N.T., Khondker, M., Kabir, S.M.H., Ahmad, M., Ahmed, A.T.A., Rahman, A.K.A. and Haque, E.U. (eds.). 2008a. Encyclopedia of Flora and Fauna of Bangladesh, Vol. 6. Angiosperms: Dicotyledons (Acanthaceae- Asteraceae). Asiatic Society of Bangladesh, Dhaka. pp. 1408.

Ahmed, Z.U., Hassan, M.A., Begum, Z.N.T., Khondker, M., Kabir, S.M.H., Ahmad, M., Ahmed, A.T.A., Rahman, A.K.A. and Haque, E.U. (eds.). 2008b. Encyclopedia of Flora and Fauna of Bangladesh, Vol. 7. Angiosperms: Dicotyledons (Balsaminaceae- Euphorbiaceae). Asiatic Society of Bangladesh, Dhaka. pp. 1 - 546.

Ahmed, Z.U., Hassan, M.A., Begum, Z.N.T., Khondker, M., Kabir, S.M.H., Ahmad, M., Ahmed, A.T.A., Rahman, A.K.A. and Haque, E.U. (eds.). 2009a. Encyclopedia of Flora and Fauna of Bangladesh, Vol. 8. Angiosperms: Dicotyledons (Fabaceae-Lythraceae). Asiatic Society of Bangladesh, Dhaka. pp. 1- 478.

Ahmed, Z.U., Hassan, M.A., Begum, Z.N.T., Khondker, M., Kabir, S.M.H., Ahmad, M. and Ahmed, A.T.A. (eds.). 2009b. Encyclopedia of Flora and Fauna of Bangladesh, 9. Angiosperms: Dicotyledons (Magnoliaceae-Punicaceae). Asiatic Society of Bangladesh, Dhaka. pp. 1- 488.

Ahmed, Z.U., Hassan, M.A., Begum, Z.N.T., Khondker, M., Kabir, S.M.H., Ahmad, M. and Ahmed, A.T.A. (eds.). 2009c. Encyclopedia of Flora and Fauna of Bangladesh, Vol. 10. Angiosperms: Dicotyledons (Ranunculaceae-Zygophyllaceae). Asiatic Society of Bangladesh, Dhaka. pp. 1-580.

Brumitt, R.K. 1992. Vascular plants families and genera, Royal Botanic Garden. England.

Brummitt, R.K. and Powell, C.E. 1992. Authors of Plant names. Royal Botanic Garden, KEW, England. pp.1- 732

Cowan, J.M. 1926. The flora of Chakaria Sundarbans. Rec. Bot. Surv. Ind. 11. pp. 997-225.

Cronquist, A. 1981. An integrated system of classification of flowering plants. Columbia University Press, Columbia.

Hajra, P.K., Nair, V.J. and Daniel, P. 1997. Flora of India 4:1-552, Botanical Survey of India, Culcutta.

Hajra, P.K. 1997. Flora of West Bengal 1:1- 486 (Flora of India Series-2). Botanical Survey of India, Calcutta.

Hara, H. and Williams, L.H.J. 1979. An enumeration of the flowering palnts of Nepal 2: 1 - 220. British Museum Natural History, London. 
Heinig, R.L. 1925. List of Plants of Chittagong collectorate and the Hill Tracts: The Bengal Government Branch Press, Darjeeling, India. pp. 1-84.

Hooker, J.D. 1872-1875. The Flora of British India I: 1-740. L. Reeve \& Co. Ltd., Kent, England. Indian reprint 1973. Bishen Sing Mahendra Pal Singh, Dhera Dun. India.

Internet sources:

(i) http://www.ars-grin.gov/cgi-bin/npgs/html/taxgenform.pl

(ii) http://epic.kew.org/searchepic/searchpage.do

(iii) http://zipcodezoo.com/Plants/D/Dalhousiea_bracteata/

(iv) http://globalnames.org/name_strings? Search_term=prodalaria+bracteata

(v) www. worldmap.asia.com

(vi) Google search: J. D. Hooker and others

Khan, M.S. (eds.), 1972-1987. Flora of Bangladesh, vols. 9, 23, 25, 26 and 36. Bangladesh National Herbarium, Dhaka. Banghadesh.

Khan, M.S. and Rahman, M. M. (eds.) 1989-2002. Flora of Bangladesh, vols. 51 and 52. Bangladesh National Herbarium, Dhaka.

Khanam, M. and Ara, H. 2007. Flora of Bangladesh, vols. 56 and 57. Bangladesh National Herbarium, Dhaka.

Kurz, W.S. 1877. Forest Flora of British Burma, 2:1-550. Reprt.,1974. Bishen Sing Mahendra Pal Singh, Dhera Dun. India.

Grierson, A.J.C., and Long, D.G. 1984. Flora of Bhutan.Vol. I Part 2. 1-462., Royal Botanic Garden, Edinburgh.

Grierson, A.J.C., and Long, D.G. 1991. Flora of Bhutan.Vol. 2 Part 1. 1-426., Royal Botanic Garden, Edinburgh.

Mabberley, D.J. 1997. The Plants - Book, a portable dictionary of the vuscular plants. (second edition) Cambridge University Press, Cambridge, U.K. pp. 1-858.

Prain, D. 1903. Bengal plants, I: 1-490; II: 491-1013. (Indian Reprint 1963). Botanical Survey of India, Bishen Singh Mahendra Singh, Dehra Dun, India.

Press, J.R., Shrestha, K.K. and Sutton, D.A. 2000. Annotated Checklist of the Flowering Plants of Nepal. The Natural History of Museum, London. pp. 1-430.

Rahman, M.A., Rashid, E. and Wilcock, C.C. 2003. Diversity, distribution and ethnobotany of the Vitaceae of Bangladesh. J. Saudi Soc. Agric. Sci. 2(1): pp.15-35.

Roxburgh, W. 1814. Hortus Bengalensis. Boerhaave press, Leiden (Holland). pp.1-105.

Roxburgh, W. 1832. Flora Indica or Description of Indian Plants, 2: 1-763. Mission press, Serampore, Calcutta, India.

Sharma, B.D., Balakrishnan, N.P. Rao, R.R. and Hajra, P.K. 1993. Flora of India, 1: 1-467. Botanical Survey of India, Culcutta.

Sharma, B.D. and Balakrishnan, N.P.1993. Flora of India, 2: 1-624. Botanical Survey of India, Culcutta.

Sharma, B.D. and Sanjappa, M. 1993. Flora of India Vol. 3: 1- 639. Botanical Survey of India, Culcutta.

Sinclair, J. 1956. The Flora of Cox’s Bazar, East Pakistan. Bull. Bot. Soc.Beng. 9(2): 1-116. Botanical Society of Bengal, Calcatta, India.

Voss, E.G. (ed.) 1983. International Code of Botanical Nomenclature (ICBN), Regnum Vegetabile,Vol. 97. Bohn, Scheltema \& Holkema, Utrecht.

Wallich, N. 1828-1849. Numerical list of dried specimens of plants in the East Indian Company's Museum, Ined.

(Manuscript received on 19 August 2011; revised on 23 October 2011) 